\title{
Switching suppression and enhancement of fluorescence and six-wave mixing by phase modulation
}

\author{
Zhiguo Wang, Peng Ying, Peiying Li, Dan Zhang, Heqing Huang, Hao Tian \& Yanpeng Zhang
}

Received

9 September 2013

Accepted

19 November 2013

Published

4 December 2013

Correspondence and requests for materials should be addressed to Y.P.Z. lypzhang@mail. xjtu.edu.cn)
Key Laboratory for Physical Electronics and Devices of the Ministry of Education \& Shaanxi Key Lab of Information Photonic Technique, Xi'an Jiaotong University, $X_{i}^{\prime}$ an 710049, China.

The conversion between enhancement and suppression in six-wave mixing (SWM) and fluorescence signals by phase modulation has demonstrated for the first time. It is observed in our experiment the suppression of SWM and fluorescence is transformed into enhancement in company with the switch from electromagnetically induced transparency (EIT) to electromagnetically induced absorption (EIA) in the transmitted probe with the relative phase changed from 0 to $\pi / 2$. Our research could be potentially applied in optical communication and quantum information processing.

ots of studies focus on the electromagnetically induced transparency (EIT) and the higher-order nonlinear optical process comprising four-wave mixing (FWM) and six-wave mixing (SWM) under EIT condition ${ }^{1-3}$. In the meantime, the fluorescence owing to spontaneous emission is also observed ${ }^{4-6}$. Moreover, the enhancement and suppression of FWM (SWM) and fluorescence corresponding to electromagnetically induced absorption (EIA) and EIT has aroused much interest ${ }^{7,8}$. The switch between bright state (EIA in the transmitted probe signal and enhancement in FWM and fluorescence signals) and dark state (EIT in the transmitted probe signal and suppression in FWM and fluorescence signals) can be realized by controlling phase difference between the two circularly polarized components of a single coherent field'. In addition, the switch can also be obtained by manipulating the dressing field power and the probe detuning ${ }^{8,10-12}$.

In this paper, we experimentally demonstrate the phase-modulated switch between enhancement and suppression of SWM and fluorescence signals for the first time. First, the phase modulated switch between bright and dark states is realized under self-dressing condition. Second, we study the phase modulated switch under multidressing condition including the external-dressing. At last, we observe the dependence of the measured signals on relative phase.

\section{Results}

Our experiment is carried out in a ${ }^{85} \mathrm{Rb}$ vapor cell. The energy levels $5 S_{1 / 2}(F=3), 5 S_{1 / 2}(F=2), 5 P_{3 / 2}, 5 D_{3 / 2}$, and $5 D_{5 / 2}$ constitute a five-level atomic system(Fig. $\left.1(\mathrm{a})\right)$. The transition $5 S_{1 / 2}(F=3)(|0\rangle)-5 P_{3 / 2}(|1\rangle)$ is probed by the laser beam $E_{1}$ (frequency $\omega_{1}$, wave vector $\mathbf{k}_{1}$ and Rabi frequency $G_{1}$ ). The transition $5 P_{3 / 2}-5 S_{1 / 2}(F=2)(|3\rangle$ ) is driven by two coupling beams $E_{3}\left(\omega_{3}, \mathbf{k}_{3}\right.$ and $\left.G_{3}\right)$ and $E_{3}^{\prime}\left(\omega_{3}, \mathbf{k}_{3}^{\prime}\right.$ and $\left.G_{3}^{\prime}\right)$. Two dressing beams $E_{2}\left(\omega_{2}, \mathbf{k}_{2}\right.$ and $\left.G_{2}\right)$ and $E_{4}\left(\omega_{4}, \mathbf{k}_{4}\right.$ and $\left.G_{4}\right)$ respectively drive the upper transitions $5 P_{3 / 2}-5 D_{5 / 2}(|2\rangle)$ and $5 P_{3 / 2}-5 D_{3 / 2}(|4\rangle)$. In normal experiment, the five beams are placed in a square-box pattern (Fig. 1(b)). The beams $E_{2}, E_{4}, E_{3}$ and $E_{3}^{\prime}$ propagate through the cell in the same direction with tiny angles about $0.3^{\circ}$ between any two. In the opposite direction of $E_{2}$ there is the probe beam $E_{1}$. However, in our experiment, the normal experimental configuration should be modified since we study the phase-controlled switch. The coupling beam $E_{2}\left(E_{4}\right)$ is deviated with an angle $\alpha(\beta)$ from the normal position (Fig. 1(c) and 1(d)). In the experimental system, we use $E_{S 1}$ and $E_{\mathrm{S} 2}$ to represent the SWM signals generated by $E_{1}, E_{3}, E_{3}^{\prime}$ and $E_{2}$ and by $E_{1}, E_{3}, E_{3}^{\prime}$ and $E_{4}$, respectively. Besides, the singlephoton fluorescence caused by the photon decay from the level $|1\rangle$ is called $R_{0}$. The sign $R_{1}\left(R_{2}\right)$ denotes the fluorescence due to the photon decay from the level $|2\rangle(|4\rangle)$.

Generally, we can obtain the density matrix elements $\rho_{10}^{(1)}$ (related with EIT), $\rho_{S 1}^{(5)}$ (related with SWM signal), and $\rho_{11}^{(2)}, \rho_{11}^{(2)}, \rho_{22}^{(4)}$ and $\rho_{33}^{(4)}$ (related with fluorescence $R_{0}, R_{1}$ and $R_{2}$ ) by solving the density-matrix equations. With Liouville pathway $\rho_{00}^{(0)} \stackrel{E_{1}}{\longrightarrow} \rho_{10}^{(1)}$ and $E_{4}$ blocked, we can obtain 

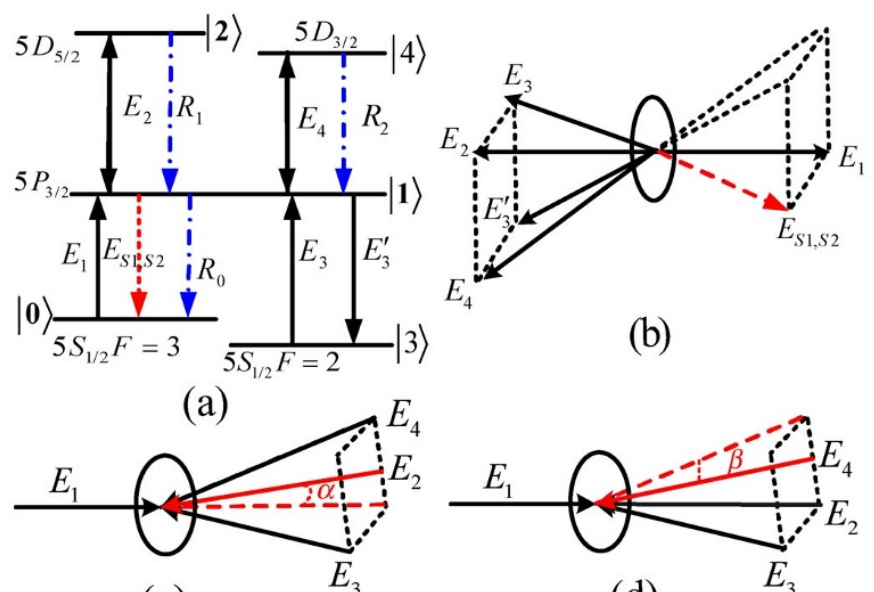

(c)

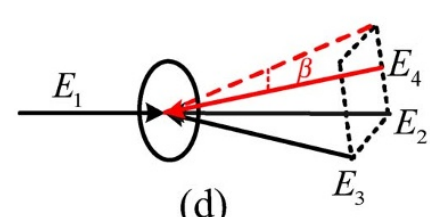

(d)

is the self-dressing term) and external dressing (the external-dressing effect refer to that the field will dresses the involved energy levels to modify the signals, while it is not the participated field for the generation of these signals, for example, the term $\left|G_{2}\right|^{2} e^{i \Delta \Phi_{1}} / d_{2}$ is the external dressing term) condition, respectively. For the fluorescence signals, via the Liouville pathway $\rho_{00}^{(0)} \stackrel{E_{1}}{\rightarrow} \rho_{10}^{(1)} \stackrel{\left(E_{1}\right)^{*}}{\rightarrow} \rho_{11}^{(2)}$, one can obtain

$$
\rho_{11 S}^{(2)}=-\left|G_{1}\right|^{2} /\left[\Gamma_{11}\left(d_{1}+\left|G_{2}\right|^{2} e^{i \Delta \Phi_{1}} / d_{2}\right)\right]
$$

or

$$
\rho_{11 D}^{(2)}=-\left|G_{1}\right|^{2} /\left[\Gamma_{11}\left(d_{1}+\left|G_{2}\right|^{2} e^{i \Delta \Phi_{1}} / d_{2}+\left|G_{4}\right|^{2} e^{i \Delta \Phi_{2}} / d_{4}\right)\right]
$$

to describe the profile of fluorescence $R_{0}$ under single-dressing or double-dressing case, respectively. With the pathway

$\rho_{33}^{(0)} \stackrel{E_{3}}{\rightarrow} \rho_{13}^{(1)} \stackrel{\left(E_{3}^{\prime}\right)^{*}}{\rightarrow} \rho_{11}^{(2)}$, one can acquire

Figure $1 \mid$ (a) Relevant ${ }^{85} \mathrm{Rb}$ five-level atomic system.(b) Normal phasematching configuration. (c) and (d) Abnormal configurations with the deflection angle $\alpha$ and $\beta$.

$$
\rho_{10 S}^{(1)}=i G_{1} /\left(d_{1}+\left|G_{2}\right|^{2} e^{i \Delta \Phi_{1}} / d_{2}\right)
$$

where $d_{1}=i \Delta_{1}+\Gamma_{10}, d_{2}=i\left(\Delta_{1}+\Delta_{2}\right)+\Gamma_{20}, \Delta_{i}=\Omega_{i}-\omega_{i}\left(\Omega_{i}\right.$ denotes the level resonance frequency and $\Gamma_{i j}$ denotes the transverse relaxation rate between states $|i\rangle$ and $|j\rangle)$. Significantly, the phase factor $e^{i \Delta \Phi_{1}}\left(\Delta \Phi_{1}\right.$ is related with the orientations of induced dipole moments $\mu_{1}$ and $\mu_{2}{ }^{12}$ ) has been introduced into the dressing term $\left|G_{2}\right|^{2} / d_{2}$ in $\rho_{10 S}^{(1)}$ since the angle $\alpha$ between $E_{2}$ and the opposite direction of $E_{1}$ is considered. With all five beams including $E_{4}$ turned on, $\rho_{10 S}^{(1)}$ is modified as

$$
\rho_{10 D}^{(1)}=i G_{1} /\left(d_{1}+\left|G_{2}\right|^{2} e^{i \Delta \Phi_{1}} / d_{2}+\left|G_{4}\right|^{2} e^{i \Delta \Phi_{2}} / d_{4}\right)
$$

with $d_{4}=i\left(\Delta_{1}+\Delta_{4}\right)+\Gamma_{40}$, where $\Delta \Phi_{2}$ is the phase factor related with the angle $\beta$. Via the Liouville pathway $\stackrel{G_{1}}{\rho_{00}^{(0)}} \rightarrow \rho_{10}^{(1)} \stackrel{\left(G_{3}^{\prime}\right)^{*}}{\rightarrow} \rho_{30}^{(2)} \stackrel{G_{3}}{\rightarrow} \rho_{10}^{(3)} \stackrel{G_{2}}{\rightarrow} \rho_{20}^{(4)} \stackrel{G_{2}^{*}}{\rightarrow} \rho_{10}^{(5)}$, the SWM signal $E_{S 1}$ can

be obtained as

$$
\rho_{S 1}^{(5)}=G_{A} e^{i \mathbf{k}_{S 1} \cdot \mathbf{r}} /\left[d_{3}\left(d_{1}+\left|G_{2}\right|^{2} e^{i \Delta \Phi_{1}} / d_{2}\right)^{3}\left(d_{2}+\left|G_{1}\right|^{2} / d_{5}\right)\right]
$$

with $E_{4}$ blocked or

$\rho_{D 1}^{(5)}=G_{A} e^{i \mathrm{k}_{S 1} \cdot \mathrm{r}} /$

$\left[d_{3}\left(d_{1}+\left|G_{1}\right|^{2} / d_{6}+\left|G_{2}\right|^{2} e^{i \Delta \Phi_{1}} / d_{2}+\left|G_{4}\right|^{2} e^{i \Delta \Phi_{2}} / d_{4}\right)^{3}\left(d_{2}+\left|G_{1}\right|^{2} / d_{5}\right)\right]$

with all five beams turned on, where $G_{A}=i G_{1} G_{3} G_{3}^{\prime *} G_{2} G_{2}^{*}, d_{3}=i\left(\Delta_{1}\right.$ $\left.-\Delta_{3}\right)+\Gamma_{30}, d_{5}=\Gamma_{21}+i \Delta_{2}+\left|G_{3}\right|^{2} /\left[\Gamma_{23}+i\left(\Delta_{2}+\Delta_{3}\right)\right]$ and $d_{6}=\Gamma_{11}+\left|G_{4}\right|^{2} e^{i \Delta \Phi_{2}} /\left(\Gamma_{41}+i \Delta_{4}\right)$. Similarly, the SWM $E_{S 2}$ can be described by

$$
\rho_{S 2}^{(5)}=G_{B} e^{i \mathbf{k}_{S 2} \cdot \mathbf{r}} /\left[d_{3}\left(d_{1}+\left|G_{4}\right|^{2} e^{i \Delta \Phi_{2}} / d_{4}\right)^{3}\left(d_{4}+\left|G_{1}\right|^{2} / d_{7}\right)\right]
$$

with $G_{B}=i G_{1} G_{3} G_{3}^{*} G_{4} G_{4}^{*}$ and $d_{7}=\Gamma_{41}+i \Delta_{4}+\left|G_{3}\right|^{2} /\left[\Gamma_{43}+i\left(\Delta_{4}+\right.\right.$ $\left.\left.\Delta_{3}\right)\right]$ and

$\rho_{D 2}^{(5)}=G_{B} e^{i \mathrm{k}_{S 2} \cdot \mathrm{r}} /$

$\left[d_{3}\left(d_{1}+\left|G_{1}\right|^{2} / d_{8}+\left|G_{2}\right|^{2} e^{i \Delta \Phi_{1}} / d_{2}+\left|G_{4}\right|^{2} e^{i \Delta \Phi_{2}} / d_{4}\right)^{3}\left(d_{4}+\left|G_{1}\right|^{2} / d_{7}\right)\right]$

with $d_{8}=\Gamma_{11}+\left|G_{2}\right|^{2} e^{i \Delta \Phi_{1}} /\left(\Gamma_{21}+i \Delta_{2}\right)$ under self-dressing (the selfdressing effect refer to that one of the participating fields for generating SWM signals dresses the involved energy levels, which then modifies the SWM process itself, for example, the term $\left|G_{4}\right|^{2} e^{i \Delta \Phi_{2}} / d_{4}$

$$
\rho_{11}^{\prime(2)}=-\left|G_{3}\right|^{2} /\left[\Gamma_{11}\left(\Gamma_{13}+i \Delta_{3}\right)\right]
$$

to describe the DC background of $R_{0}$. Consequently, the fluorescence $R_{0}$ is proportional to $\left|\rho_{11 D}^{(2)}\right|^{2}+\left|\rho_{11}^{\prime(2)}\right|^{2}$. For two-photon fluorescence

$$
\begin{gathered}
R_{1} \text {, via } \rho_{00}^{(0)} \stackrel{E_{1}}{\rightarrow} \rho_{10}^{(1)} \stackrel{E_{2}}{\rightarrow} \rho_{20}^{(2)} \stackrel{\left(E_{1}\right)^{*} \rightarrow}{\rightarrow} \rho_{21}^{(3)} \stackrel{\left(E_{2}\right)^{*}}{\rightarrow} \rho_{22}^{(4)}, \text { we have } \\
\rho_{22 S}^{(4)}=\left|G_{1}\right|^{2}\left|G_{2}\right|^{2} /\left[\Gamma_{22} d_{1} d_{9}\left(d_{2}+\left|G_{2}\right|^{2} e^{i \Delta \Phi_{1}} / d_{1}\right)\right]
\end{gathered}
$$

with $d_{9}=\Gamma_{21}+i \Delta_{2}$. Similarly, the other two-photon fluorescence $R_{2}$ is described as

$$
\rho_{44 S}^{(4)}=\left|G_{1}\right|^{2}\left|G_{4}\right|^{2} /\left[\Gamma_{44} d_{1} d_{10}\left(d_{4}+\left|G_{4}\right|^{2} e^{i \Delta \Phi_{2}} / d_{1}\right)\right]
$$

with $d_{10}=\Gamma_{41}+i \Delta_{4}$.

First, we consider the phase-modulated switch of self-dressed signals as shown in Fig. 2. In the experiment, we turn on $E_{1}, E_{2}, E_{3}^{\prime}, E_{3}$ and block $E_{4}$ and the signals are obtained by scanning $\Delta_{2}$ at different discrete designated $\Delta_{1}$ with $\Delta \Phi_{1}=0$ (Fig. 2(a), viewed as the reference point at the normal configuration), $\Delta \Phi_{1}=-\pi / 5$ (Fig. 2(b)) and $\Delta \Phi_{1}=3 \pi / 5$ (Fig. 2(c)). In Fig. 2(a1), the EIT caused by $E_{2}$, meeting $\Delta_{1}+\Delta_{2}=0$, emerges in the larger range of the probe detuning. The EIA, satisfying $\Delta_{1}+\Delta_{2}=\left|G_{2}\right|^{2} / \Delta_{1}$, only appears at the large probe detuning, such as $\Delta_{1}= \pm 400 \mathrm{MHz}$. In Fig. 2(b1), we can find the probe transmission signals present as EIT at negative detunings $\Delta_{1}$ while change from strong EIT to partial-EIT-partialEIA and lastly to week EIA with $\Delta_{1}$ increasing at positive detunings $\Delta_{1}$. In Fig. 2(c1), with the probe detuning transformed from negative to positive, the signals turn from EIA to partial-EIT-partial-EIA and lastly to EIT. Obviously, the variations of the probe transmission signals are quite the contrary in above three figures. This is caused by the modulation of the relative phase $\Delta \Phi_{1}$ in the dressing term $\left|G_{2}\right|^{2} e^{i \Delta \Phi_{1}} / d_{2}$ in $\rho_{10 D D}^{(1)}$. Thus, at a certain detuning $\Delta_{1}$, we can switch EIT and EIA by adjusting the relative phase $\Delta \Phi_{1}$. Similarly, the dressing effect on the SWM signal caused by $\left|G_{2}\right|^{2} e^{i \Delta \Phi_{1}} / d_{2}$ is also regulated by $\Delta \Phi_{1}$. According to $\rho_{S 1}^{(5)}$, the intensity of SWM has inverse correlation with $\cos \left(\Delta \Phi_{1}-\theta\right)$ where $\theta=\arctan \left(\Delta_{1} / \Gamma_{10}\right)$. In the SWM signals, with the relative phase $\Delta \Phi_{1}$ changed from 0 (Fig. 2(a2)) to $-\pi / 5$ (Fig. 2(b2)), the intensity of SWM is obviously enhanced at $\Delta_{1} \geq 0$ since $\cos (-\pi / 5-\theta)<\cos (0-\theta)$ in the region while is suppressed at $\Delta_{1}<0$ for $\cos (-\pi / 5-\theta)>\cos (0-\theta)$ here. One can also see the difference between the SWM signals with $\Delta \Phi_{1}=$ 0 (Fig. 2(a2)) and those with $\Delta \Phi_{1}=3 \pi / 5$ (Fig. 2(c2)). Compared with the SWM in Fig. 2(a2), the SWM signal in Fig. 2(c2) is suppressed in 


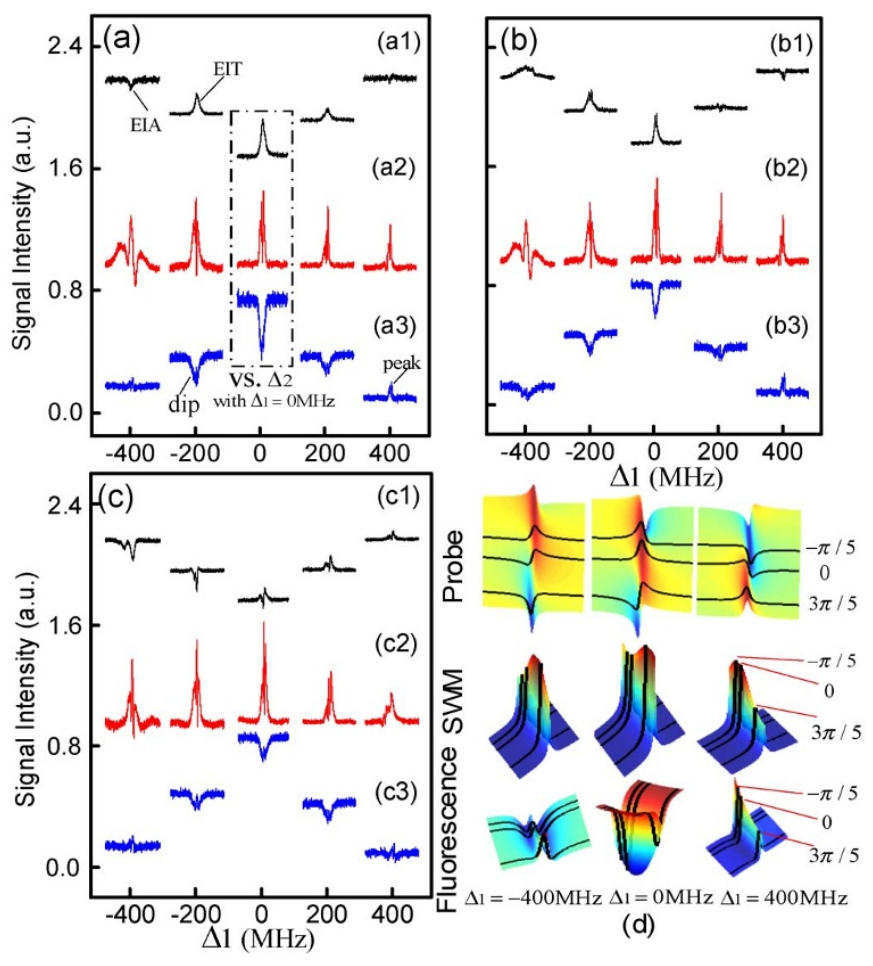

Figure $2 \mid$ In each sub-curve, measured probe transmission signals ((a1), (b1) and (c1)), SWM signals ((a2), (b2) and (c2)) and fluorescence signals ((a3), (b3) and (c3)) versus $\Delta_{2}\left(\Delta_{2}\right.$ is scanned around $-\Delta_{1}$ from $-90 \mathrm{MHz}$ to $90 \mathrm{MHz}$ ) with $E_{4}$ blocked.Each sub-curve corresponds to different fixed $\Delta_{1}$. For (a1)-(a3) $\Delta \Phi_{1}=0$, for (b1)-(b3) $\Delta \Phi_{1}=-\pi / 5$ and for (c1)-(c3) $\Delta \Phi_{1}=3 \pi / 5$. The other parameters are $\Delta_{3}=0, G_{1}=10.85 \mathrm{MHz}, G_{2}=$ $19.46 \mathrm{MHz}, G_{3}=16.66 \mathrm{MHz}$ and $G_{3}^{\prime}=14.4 \mathrm{MHz}$. (d) Theoretical calculations for probe, SWM and fluorescence signals versus $\Delta \Phi_{1}$ by scanning $\Delta_{2}$ at three typical detunings $\Delta_{1}$.

the region $\Delta_{1} \geq 200 \mathrm{MHz}$ and enhanced in the region $\Delta_{1}<$ $200 \mathrm{MHz}$. From Fig. 2(a3) to Fig. 2(b3), one can find, with EIT converted to EIA (for example, at $\Delta_{1}=200 \mathrm{MHz}$ from Fig. 2(a1) to Fig. 2(b1)), on one hand, the dip of the corresponding fluorescence gets shallow due to the weakened dressing effect of $E_{2}$ on $R_{0}$ (in company with EIA) according to $\rho_{11 S}^{(2)}$, on the other hand, the peaks in the dips get high because the original suppression (in company with EIT) induced by the dressing term $\left|G_{2}\right|^{2} e^{i \Delta \Phi_{1}} / d_{2}$ in $\rho_{22 S}^{(4)}$ turns to enhancement (in company with EIA). While EIA (Fig. 2(a1)) is converted to EIT (Fig. 2(b1)), for example, at $\Delta_{1}=-400 \mathrm{MHz}$, the variations of the fluorescence signals are just the opposite. Same as above, when $\Delta \Phi_{1}$ is changed from 0 (Fig. 2 (a3)) to $3 \pi / 5$ (Fig. 2(c3)), the similar variations also appear in the fluorescence signals, for example, at $\Delta_{1}=-200 \mathrm{MHz}$ and $\Delta_{1}=400 \mathrm{MHz}$. We have simulated the EIA-EIT switch in the probe transmission signal and suppression-enhancement transformation in SWM and fluorescence process, as shown in Fig. 2(d) which agrees with the experimental results very well.

Further, we turn on all five laser beams including $E_{4}$ and observe the phase controlled switch of the multi-dressed (the multi-dressing effect is the case where two or more fields including self- and external-dressing fields dress the energy levels to modify the signals) signals by scanning $\Delta_{4}$ at different discrete fixed $\Delta_{1}$ (Fig. 3). In Fig. 3(a1), the peak higher than the baseline in each sub-curve represents the EIT induced by $E_{4}$. The global profile formed from all baselines means the EIT induced by $E_{2}$, meeting the condition $\Delta_{1}$ $+\Delta_{2}=0$. When we change the related phase $\Delta \Phi_{2}$ from 0 (Fig. 3(a1)) to $\pi / 2$ (Fig. 3(a2)), the original EITs are mostly switched to EIA. In Fig. 3(b1), the dip in each sub-curve shows the suppression on $R_{0}$

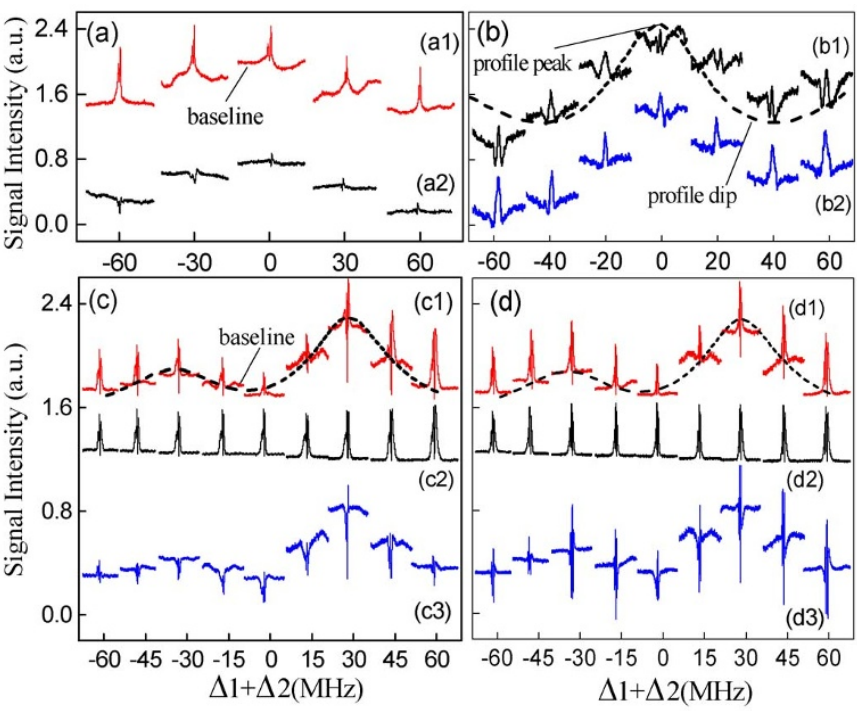

Figure $3 \mid$ In each sub-curve, measured probe transmission signals (a), fluorescence signals (b), and SWM signals (c)-(d) versus $\Delta_{4}\left(\Delta_{4}\right.$ is scanned around $-\Delta_{1}$ from $-90 \mathrm{MHz}$ to $90 \mathrm{MHz}$ ) with the fixed $\Delta_{2}$. Each sub-curve corresponds to different discrete fixed $\Delta_{1}$. (a1), (b1) and (c) Signals obtained with $\Delta \Phi_{2}=0$. (a2), (b2) and (d) Signals obtained with $\Delta \Phi_{2}=\pi / 2$. (a), (b), (c1) and (d1) Signals obtained with all beams turned on. (c2) and (d2) Signals obtained with $E_{2}$ blocked. (c3) Signals obtained by subtracting the signals in (c2) from the signals in (c1). (d3) Signals obtained by subtracting the signals in (d2) from the signals in (d1). Other parameters are $\Delta_{2}=100 \mathrm{MHz}, \Delta_{3}=0, \Delta \Phi_{1}=0, G_{1}=10.72 \mathrm{MHz}, G_{2}=$ $21.16 \mathrm{MHz}, G_{3}=18.87 \mathrm{MHz}, G_{3}^{\prime}=14.4 \mathrm{MHz}$ and $G_{4}=14.16 \mathrm{MHz}$.

caused by the dressing field $E_{4}$ and the peak in each dip represents the two-photon fluorescence $R_{2}$. The profile dip consisting of baselines reveals $R_{0}$ suppressed by $E_{2}$ owing to the dressing term $\left|G_{2}\right|^{2} / d_{2}$ in $\rho_{11 D}^{(2)}$ and the profile peak represents the two-photon fluorescence $R_{1}$. With $\Delta \Phi_{2}$ changed from 0 (Fig. 3(b1)) to $\pi / 2$ (Fig. 3(b2)), the height of the peak in each sub-curve gets much high because the dressing term $\left|G_{4}\right|^{2} e^{i \Delta \Phi_{2}} / d_{1}$ in $\rho_{44 S}^{(4)}$ has generated the enhancement effect on $R_{2}$ at $\Delta \Phi_{2}=\pi / 2$. In this process, the dip in each sub-curve becomes shallow due to the weakened dressing effect of $E_{4}$ on $R_{0}$ caused by the modulation of $\Delta \Phi_{2}$.

In Fig. 3(c1), the global profile (dashed curve) consisting of all baselines reveals the SWM signal $E_{S 1}$. In the $E_{S 1}$ signal, one can find the AT splitting which is because the self-dressing term $\left|G_{1}\right|^{2} / d_{5}$ acts on the two-photon term $d_{2}$ in $\rho_{D 1}^{(5)}$. The sub-curve at any $\Delta_{1}$ means the compound signal which includes two components: $I_{D 1}$ and $I_{D 2} . I_{D 1}$ denotes the enhancement or suppression intensity of $E_{S 1}$ (arising from the external-dressing field $E_{4}$ according to $\left.\rho_{D 1}^{(5)}\right)$ and $I_{D 2}$ signifies the $S W M E_{S 2}$ intensity with the suppression of $E_{2}$. Figure 3(c2) shows the SWM $E_{S 2}$ intensity without the suppression of $E_{2}$ which is denoted by $I_{S 2}$. The signals in Fig. 3(c3) are obtained by subtracting the signals $\left(I_{S 2}\right)$ in Fig. 3(c2) from the signals $\left(I_{D 1}+I_{D 2}\right)$ in Fig. 3(c1). Therefore, the signals in Fig. 3(c3) represent two dressing results at different $\Delta_{1}$ : one is $I_{D 1}$, the other is $I_{D 2}-I_{S 2}$ which is the suppressed intensity caused by $E_{2}$ with regard to the $S W M E_{S 2}$. When we alter the relative phase $\Delta \Phi_{2}$, the experiment results similar to Fig. 3(c) are obtained, as shown in Fig. 3(d1)-3(d3). The modulation of signals caused by relative phase can be observed clearly, as shown in Fig. 3(c3) and Fig. 3(d3). For instance, with the relative phase switched from 0 to $\pi / 2$, the original suppression signals (at $\Delta_{1}+$ $\Delta_{2}=-45,-30$ and $-15 \mathrm{MHz}$ ) transform into partial-suppressionpartial-enhancement signals and the intensities of original partialsuppression-partial-enhancement signals (at $\Delta_{1}+\Delta_{2}=0,15,30,45$ and $60 \mathrm{MHz}$ ) are strengthened significantly in correspondence with 
the EIT-EIA switch in Fig. 3(a). It is worth mentioning that the dressing result of $E_{2}$ is basically invariable since the relative phase $\Delta \Phi_{2}$ is only related to the dressing effect of $E_{4}$.

\section{Discussion}

In order to clearly compare the variations of signals with the relative phase, we discuss the relative phase dependence of the measured signals by scanning $\Delta_{4}$ at the fixed detuning $\Delta_{1}$ under the above mentioned two cases of self-dressing and multi-dressing effects. First, with $E_{2}$ blocked (Fig. 4(a), the self-dressing case), EIT in Fig. 4(a1) can be switched to EIA along with the change of $\Delta \Phi_{2}$ from bottom to top. During this process, the strongest EIT and EIA separately appear at $\Delta \Phi_{2}=-\pi / 6$ and $\Delta \Phi_{2}=3 \pi / 4$. Depending on whether $\Delta \Phi_{2}$ is greater than or less than $\pi / 4$, the transmitted probe signal behaves mainly EIA or EIT. In the phase matching range where $\Delta \Phi_{2}$ is altered from $-\pi / 6$ to $\pi / 2$, compared with the signals at the reference phase $\Delta \Phi_{2}=0$, the SWM signal $E_{S 2}$ (Fig. 4(a2)) and fluorescence signal $R_{2}$ (peaks in Fig. 4(a3)) get large when $\Delta \Phi_{2}>0$ and become small while $\Delta \Phi_{2}<0$ due to the switch of the dressing effect of $E_{4}$ induced by $\Delta \Phi_{2}$. The largest intensities of the SWM and fluorescence signals appear at partial-EIT-partial-EIA $\left(\Delta \Phi_{2}=\pi / 4\right)$ and EIA $\left(\Delta \Phi_{2}=\pi / 2\right)$, respectively. Along with continuing to increase $\Delta \Phi_{2}$ from $\pi / 2$ to $\pi$ or to decrease $\Delta \Phi_{2}$ from $-\pi / 6$ to $-\pi$ / 3 , which correspond to increasing the deflection angle $\beta$ under the abnormal configuration (Fig. 1(d)), the SWM and fluorescence signal intensities decrease. This results from the classical effect with the
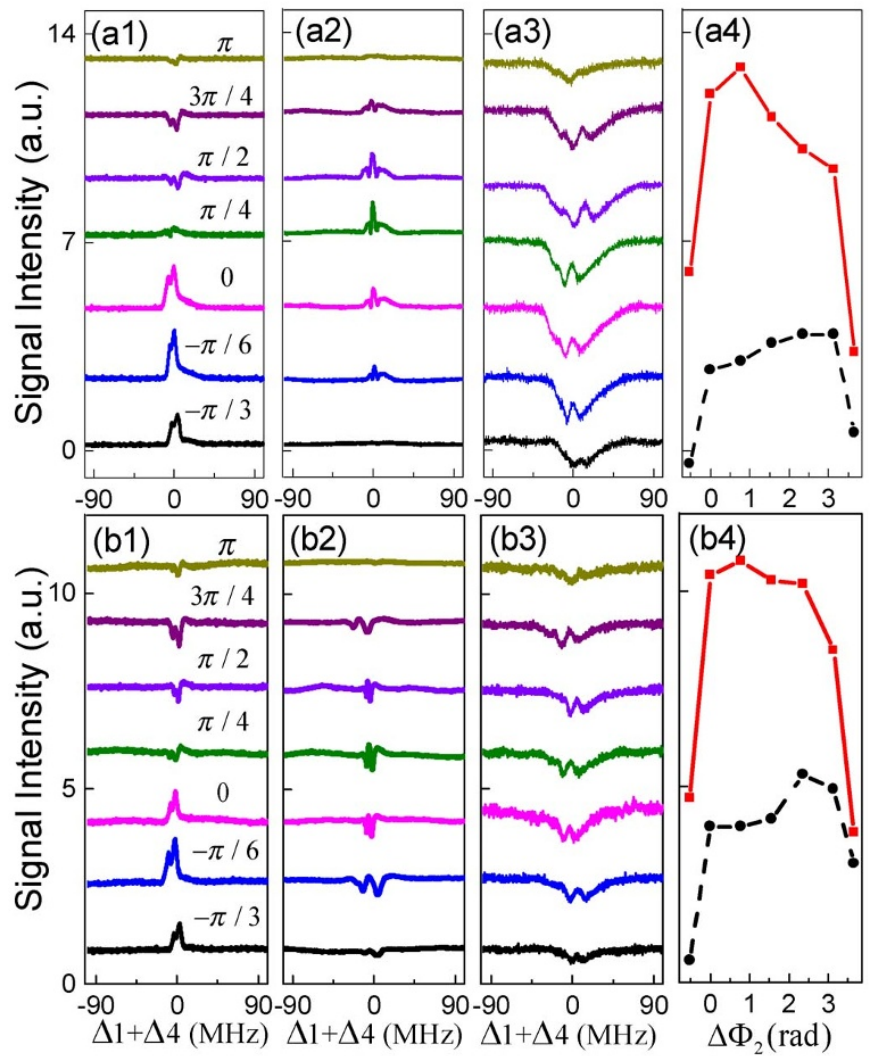

Figure $4 \mid$ The transmitted probe ((a1), (b1)), SWM ((a2), (b2)) and fluorescence ((a3), (b3)) versus $\Delta_{4}$ at different relative phase $\Delta \Phi_{2}=-\pi / 3$, $-\pi / 6,0, \pi / 4, \pi / 2,3 \pi / 4$, and $\pi$ from the bottom to top.(a1),(a2) and (a3) Signals obtained with $E_{2}$ blocked. (b1),(b2) and (b3) Signals obtained with all beams turned on. (a4) and (b4) the relative phase dependences of the fluorescence peak (dash line with circles) and the fluorescence dip (solid line with squares) in (a3) and (b3), respectively. The other parameters are $\Delta \Phi_{1}=0, \Delta_{1}=-120 \mathrm{MHz}, \Delta_{2}=150 \mathrm{MHz}, \Delta_{3}=0, G_{1}=11.84 \mathrm{MHz}, G_{3}$ $=18.34 \mathrm{MHz}, G_{3}^{\prime}=17 \mathrm{MHz}, G_{2}=26.11 \mathrm{MHz}$ and $G_{4}=18 \mathrm{MHz}$. phase mismatching condition. For the sake of clarity, the relative phase dependence of the fluorescence peak $R_{2}$ is also shown by dash line with circles in Fig. 4(a4). The fluorescence suppression dip (Fig. 4(a3)) induced by $E_{4}$ evolves from shallow to deep, then to shallow, the variation of which with the relative phase is also illustrated by the solid line with squares in Fig. 4(a4). The fluorescence suppression dip reaches the deepest at the stronger EIT. Next, with all five beams on (Fig. 4(b), the multi-dressing case), the evolvement of probe transmission signal in Fig. 4(b1) is similar to that in Fig. 4(a1) except that the EIT peak in Fig. 4(b1) is weaker than the one in Fig. 4(a1) due to the strong cascade-dressing interaction between $E_{2}$ and $E_{4}$. The peaks higher than the baselines in Fig. 4(b2) show the variation of the intensity $I_{D 2}$ of SWM signal $E_{S 2}$ under the dressing effect of $E_{2}$, the evolvement of which approximates to that of the SWM peaks in Fig. 4(a2). The dips lower than the baselines in Fig. 4(b2) illustrate the variation of the suppression signal intensity $I_{D 1}$ of $S W M E_{S 1}$. In the phase matching range, the suppression dips of SWM $E_{S 1}$ are relatively deep under the condition of EIT and become shallow in the case of EIA. The variations of fluorescence peak $R_{2}$ and fluorescence suppression dip in Fig. 4(b3), the relative phase dependences of which are shown in Fig. 4(b4), accord with those in Fig. 4(a3) except that the peaks become slightly small and the dips get shallow because of the cascade-dressing interaction between $E_{2}$ and $E_{4}$.

In summary, we study the phase modulated switch between suppression and enhancement of fluorescence and SWM. By manipulating the relative phase, the original suppression on SWM and fluorescence signals can be transformed into enhancement in company with the conversion from EIT to EIA in the transmitted probe. Such switch can be applied in optical communication and quantum information processing.

\section{Methods}

In our experiment, there are five laser beams generated by four external cavity diode lasers (ECDL) with linewidths of less than or equal to $1 \mathrm{MHz}$. The probe laser beam $E_{1}$ is from an ECDL with wavelength of $780.245 \mathrm{~nm}$ and a horizontal polarization. The two coupling laser beams $E_{3}$ and $E_{3}^{\prime}$ with wavelength $780.235 \mathrm{~nm}$ and a vertical polarization are split from another ECDL. The dressing laser beam $E_{2}\left(E_{4}\right)$ with wavelength of $775.978 \mathrm{~nm}(776.157 \mathrm{~nm})$ and a vertical polarization are from other two ECDLs. The atomic vapor cell temperature is set at $60^{\circ} \mathrm{C}$, corresponding to the typical density of $2 \times 10^{11} \mathrm{~cm}^{-3}$. In our five-level atomic system, there exist two ladder-type EIT windows, two SWM signals and three fluorescence signals under the normal experimental configuration (Fig. 1(b)). Specifically, one SWM signal $E_{S 1}$ is generated by $E_{1}, E_{3}, E_{3}^{\prime}$, and $E_{2}$ in the $|0\rangle-|1\rangle-|2\rangle$ ladder-type EIT window, which satisfies the phase matching condition $\mathbf{k}_{S 1}=\mathbf{k}_{1}+\mathbf{k}_{3}-\mathbf{k}_{3}^{\prime}+\mathbf{k}_{2}-\mathbf{k}_{2}$, and the other SWM signal $E_{S 1}$ is generated by $E_{1}, E_{3}, E_{3}^{\prime}$ and $E_{4}$ in the $|0\rangle-|1\rangle-|4\rangle$ ladder-type EIT window, which satisfies the phase matching condition $\mathbf{k}_{S 2}=\mathbf{k}_{1}+\mathbf{k}_{3}-\mathbf{k}_{3}^{\prime}+\mathbf{k}_{4}-\mathbf{k}_{4}$. Besides, the single-photon fluorescence $R_{0}$ is caused by the photon decay from the level $|1\rangle$. The two-photon fluorescence $R_{1}$ and $R_{2}$ are due to the photon decay from the level $|2\rangle$ and $|4\rangle$, respectively. When the coupling beam $E_{2}\left(E_{4}\right)$ is deviated with an angle $\alpha(\beta)$ from the normal position (Fig. 1(c) and 1(d)), the above EIT windows, SWM and fluorescence signals will switch between bright state (EIA in the transmitted probe signal and enhancement in SWM and fluorescence signals) and dark state (EIT in the transmitted probe signal and suppression in SWM and fluorescence signals).

1. Wielandy, S. \& Gaeta, A. Investigation of electromagnetically induced transparency in the strong probe regime. Phys. Rev. A 58, 2500-2505 (1998).

2. Kash, M. et al. Ultraslow group velocity and enhanced nonlinear optical effects in a coherently driven hot atomic gas. Phys. Rev. Lett. 82, 5229-5232 (1999).

3. Ham, B. S., Shahriar, M. S. \& Hemmer, P. R. Enhanced nondegenerate four-wave mixing owing to electromagnetically induced transparency in a spectral holeburning crystal. Opt. Lett. 22, 1138-1140 (1997).

4. Harris, S. E. Electromagnetically induced transparency. Phys, Today 50, 36-42 (1997).

5. Qi, J. et al. Autler-Townes splitting in molecular lithium: Prospects for all-optical alignment of nonpolar molecules. Phys. Rev. Lett. 83, 288-291 (1999).

6. Qi, J. \& Lyyra, A. M. Electromagnetically induced transparency and dark fluorescence in a cascade three-level diatomic lithium system. Phys. Rev. A, 73, 043810 (2006).

7. Akulshin, A. M., Barreiro, S. \& Lezama, A. Electromagnetically induced absorption and transparency due to resonant two-field excitation of quasidegenerate levels in Rb vapor. Phys. Rev. A 57, 2996-3002 (1998). 
8. Li, N. et al. Observation of dressed odd-order multi-wave mixing in five-level atomic medium. Opt. Exp. 20, 1912-1929 (2012).

9. Khadka, U., Zhang, Y. P. \& Xiao, M. Control of multitransparency windows via dark-state phase manipulation. Phys. Rev. A, 81, 023830 (2010).

10. Li, C. et al. Observation of enhancement and suppression in four-wave mixing processes. Appl. Phys. Lett. 95, 041103 (2009).

11. Wang, Z. et al. Switching enhancement and suppression of four-wave mixing via a dressing field. J. Mod. Opt. 58, 802-809 (2011).

12. Hahn, K. H., King, D. A. \& Harris, S. E. Nonlinear generation of 104.8-nm radiation within an absorption window in zinc. Phys. Rev. Lett. 65, 2777-2779 (1990).

\section{Acknowledgments}

This work was supported by the 973 Program (2012CB921804), NSFC (61078002, 61078020, 11104214, 61108017, 11104216, 61205112), RFDP (20110201110006, 20110201120005, 20100201120031), FRFCU (2012jdhz05, 2011jdhz07, xjj2011083, xjj2011084, xjj2012080, xjj2013089), and CPSF (2012M521773).

\section{Author contributions}

Z.G.W. and Y.P.Z. provided the idea and main contributions to the theoretical and experimental analysis of this work. P.Y., P.Y.L., D.Z., H.Q.H. and H.T. contributed to the presentation and execution of the work. All authors discussed the results and contributed to writing the manuscript.

\section{Additional information}

Competing financial interests: The authors declare no competing financial interests.

How to cite this article: Wang, Z. G. et al. Switching suppression and enhancement of fluorescence and six-wave mixing by phase modulation. Sci. Rep. 3, 3417; DOI:10.1038/ srep03417 (2013).

(c) (1) (3) (2) This work is licensed under a Creative Commons Attribution-

NonCommercial-ShareAlike 3.0 Unported license. To view a copy of this license, visit http://creativecommons.org/licenses/by-nc-sa/3.0 